\title{
Cinética de remoção de matéria orgânica em sistemas alagados construídos cultivados com lírio amarelo
}

\author{
Renata C. Chagas ${ }^{1}$, Antonio T. de Matos ${ }^{2}$, Paulo R. Cecon ${ }^{3}$, \\ Paola A. V. Lo Monaco ${ }^{4} \&$ Luis G. F. França ${ }^{2}$
}

\begin{abstract}
RESU M O
O bjetivou-se, com a realização deste trabalho, obter o ajuste do modelo matemático adaptado de cinética de primeira ordem e os respectivos parâmetros cinéticos para descrição da degradação da matéria orgânica em SACs cultivados com lírio amarelo (Hemerocallis flava), no tratamento de esgoto doméstico. O s SACs foram cultivados com lírio amarel o e submetidos a diferentes taxas de carregamento orgânico superficial (TCOs) de esgoto doméstico: 44; 98; 230 e $395 \mathrm{~kg} \mathrm{ha}^{-1} \mathrm{~d}^{-1}$ de DBO 0 modelo matemático adaptado de decaimento do material orgânico apresentou bom ajuste e descreveu adequadamente a cinética de remoção de matéria orgânica (DQ O) nos sistemas avaliados apresentando, geralmente, $R^{2}$ superior a $75 \%$. Em ordem crescente de TCO valores de coeficientes de degradação $\left(K_{v}\right)$ foram, respectivamente, de 0,$45 ; 0,69 ; 1,23$ e $1,72 \mathrm{~d}^{-1}$, considerados baixos para os dois primeiros SACs e normais para os outros dois, e os valores de " $n$ " de 0,$448 ; 0,107 ; 0,327$ e 0,461 , na faixa normalmente encontrada na literatura.
\end{abstract}

Palavras-chave: wetlands construídos, plantas ornamentais, cinética de degradação

\section{O rganic matter removal kinetics in constructed wetlands cultivated with yellow lily}

\begin{abstract}
A B ST RACT
This w ork aimed to obtain the adjustment of the mathematical model adapted from the first order kinetics and its respective parameters for describing the organic matter degradation in constructed wetlands (CW s) planted with yellow lily (Hemerocall is flava) for the treatment of domestic sewage. The CW s were cultivated with yellow lily and subjected to different surface organic loading rates (TCOs) of domestic sewage: 44, 98, 230 and $395 \mathrm{~kg} \mathrm{ha}^{-1}$ day $^{-1} \mathrm{BOD}$. The mathematical model adapted from the decay of organic material showed a good fit and adequately described the kinetics of removal of organic matter (COD) in the evaluated systems, with generally more than $75 \% \mathrm{R}^{2}$. In ascending order of TCO values of degradation coefficients $(\mathrm{Kv})$ were, respectively, of $0.45,0.69,1.23$ and $1.72 \mathrm{~d}^{-1}$, considered low for the first two CW s and normal for the other two, and the values of " $n$ " of $0.448,0.107,0.327$ and 0.461 in the range normally found in the literature.
\end{abstract}

Key words: constructed wetlands, ornamental plants; degradation kinetics

\footnotetext{
${ }^{1}$ D outoranda em Engenharia Ambiental/ UFES. Fone: (27) 9812-3127. E-mail: renataeab@hotmail.com.

2 DEA/UFV. Av. PH Rolfs, s/n, Fone: (31) 3899-2729. E-mail: atmatos@ufv.br; luisgustavo2f@yahoo.com.br

${ }^{3}$ D PI/U FV. Av. PH Rolfs, s/n, Fone (31) 3899-1781. E-mail: cecon@ufv.br

${ }^{4}$ Instituto Federal de Educação, Ciência e Tecnologia do Espírito Santo. Fone: (31) 3899-1871. E-mail: paolalomonaco2004@yahoo.com.br
} 


\section{INTRODUÇÃO}

Dentre as diversas soluções propostas para o tratamento de águas residuárias ricas em material orgânico, se distingue sua disposição em Sistemas Alagados Construídos ou "constructed wetlands".

Nesses sistemas a remoção de poluentes é decorrente de mecanismos físicos, químicos e biológicos incluindo-se, dentre eles, processos de sedimentação, filtração, absorção, precipitação e adsorção química, interações microbianas, extração pelas plantas, volatilização e complexação (Wood, 1995).

A vegetação implantada nos referidos sistemas atua como extratora de macro e micronutrientes necessários ao seu crescimento, além de transferir oxigênio para o substrato permitindo a formação de sítios aeróbios em torno de rizomas e raízes. Essas plantas também favorecem o desenvolvimento dos filmes biologicamente ativos que propiciam a degradação dos compostos orgânicos, depurando o meio (Matos et al., 2009a).

Diversas espécies vegetais naturalmente adaptadas a ambientes alagados, têm sido implantadas nesses sistemas, tal como a taboa (Typha latifólia L.) (Brasil et al., 2007a; 2007b; Fia et al., 2008; Fia, 2009; Matos et al., 2009a; 2009b; Wang et al., 2009), espécies vegetais da família das gramíneas, como o capim tifton-85 (Cynodon spMatos et al., 2008; (Fia, 2009; Matos et al., 2009a; 2009b; 2010a; 2010b), o capim elefante (Pennisetum purpureum) (Matos et al., 2008), azevém (Lolium multiflorum) (Fia et al., 2010), aveia preta (Avena strigosa Schreb) (Eustáquio Júnior et al., 2010, Fia et al., 2010), além de plantas ornamentais, como a helicônia (Heliconia psittacorum) (Konnerup et al., 2009), o copo-de-leite (Zantedeschia aethiopica) (Belmont \& Metcalfe, 2003; Zurita et al., 2008; 2009), o agapanto (Agapanthus africanus) (Zurita et al., 2009) e outras. Por possuírem valor econômico, as plantas ornamentais, além de contribuir para a composição paisagística, se tornam ideais para cultivo em SACs a serem utilizados no tratamento de águas residuárias em áreas como parques, pousadas, hotéis e clubes campestres.

De acordo com Valentim (2003), em vista de sua simplicidade conceitual e facilidade de construção, pelo seu baixo consumo de energia, pela sua incorporação harmônica à paisagem, por sua versatilidade e longevidade, os leitos cultivados podem ter seu uso recomendado no tratamento de diferentes tipos de águas residuárias. Além do esgoto doméstico (Brasil et al., 2005; 2007a, 2007b; Brasil \& Matos, 2008, Konnerup et al., 2009; Zurita et al., 2009), os SACs têm sido propostos e utilizados no tratamento de diversas águas residuárias como as de suinocultura (Matos et al., 2009a; 2009b; 2010a; 2010b e Fia, 2009), laticínios (Matos et al., 2008), do processamento dos frutos e do cafeeiro (Fia et al., 2008; 2010), dentre outras.

Para Kincanon \& McAnally (2004), a capacidade de um SAC em remover poluentes é função de suas características físicas, químicas e biológicas. Referidas características e o posterior desempenho dos sistemas podem ser descritos por meio de modelos matemáticos que, por sua vez, podem auxiliar na compreensão de como se processa e estimativa da remoção de poluentes e a dinâmica desta remoção no sistema.
Os modelos mais comumente utilizados para descrição da cinética de remoção do material orgânico em SACs são as equações de primeira ordem que predizem um decaimento exponencial da concentração afluente (Rousseau et al., 2004). Adaptações a este modelo foram propostas por Kadlec \& Knight (1996), Reed et al. (1995), Laber et al. (1999), Cooper (1999), além de Brasil (2007b).

De acordo com Kincanon \& Mcanally (2004), para a aplicação do modelo de cinética de primeira ordem presume-se que as transformações químicas sejam irreversíveis, ocorrendo sob taxa de reação homogênea, além de se considerar que são sistemas estáveis com modelo hidráulico de escoamento do tipo pistão. Esta abordagem ignora a influência de fatores como precipitação, evapotranspiração e padrões de escoamento não ideal (Carleton, 2002).

Para Brasil et al. (2007b) a inadequação do modelo cinético de primeira ordem com concepção de escoamento do tipo pistão na predição de remoção de matéria orgânica se deve ao fato do coeficiente de decaimento $(\mathrm{K})$ não ser verdadeiramente uma constante ao longo do comprimento dos SACs, decrescendo do início para o final do leito de tratamento; este decréscimo é devido, provavelmente, ao aumento relativo da quantidade de matéria orgânica recalcitrante ao longo do sistema, com menor taxa de degradação, e à alteração no tempo de detenção hidráulica e na dispersão, em razão de alterações no comportamento hidrodinâmico no sistema. Assim, o modelo de remoção modificado e proposto pelos autores $\left[\mathrm{C}_{\mathrm{e}} / \mathrm{C}_{\mathrm{a}}=\exp (-\right.$ $\left.\mathrm{K}_{\mathrm{V}} \mathrm{t}^{\mathrm{n}}\right)$ ], obtido em SACs utilizados no tratamento de esgoto doméstico, leva em consideração a inserção de um novo coeficiente na equação, proporcionando melhor representatividade da cinética de degradação de matéria orgânica de águas residuárias em sistemas alagados. O modelo proposto proporcionou bom ajuste e descreveu adequadamente a cinética de remoção de carga orgânica no sistema avaliado. Todavia, os autores ressaltaram que este modelo deve ser calibrado para outras águas residuárias e em outros sistemas alagados construídos, nos quais seja estudada a magnitude da dispersão, utilizando-se traçadores e associação delas com o coeficiente $n$, para possibilitar melhores ajustes do modelo.

Objetivou-se, com a realização deste trabalho, obter o ajuste do modelo matemático adaptado de cinética da primeira ordem e os respectivos parâmetros cinéticos para descrição da degradação da matéria orgânica em SACs cultivados com lírio amarelo (Hemerocallis flava), no tratamento de esgoto doméstico.

\section{Material e MÉTODOS}

O experimento foi conduzido na Área Experimental de Tratamento de Resíduos Urbanos do Departamento de Engenharia Agrícola, Universidade Federal de Viçosa, em Viçosa, MG, altitude média de 649 m, latitude $20^{\circ} 45^{\prime} 14^{\prime \prime} \mathrm{S}$ e longitude $42^{\circ} 52^{\prime} 53^{\prime \prime} \mathrm{W}$. O esgoto doméstico, proveniente do Condomínio Bosque Acamari, foi bombeado para a área experimental, na qual recebeu tratamento preliminar, constituído por desarenador, caixa de gordura, peneira e tanque de homogeneização. A distribuição do esgoto doméstico nos SACs 
ocorreu por gravidade sendo que os afluentes aos SACs 1, 2 e 3 passaram, ainda, por tratamento primário (tanque séptico retangular de alvenaria, com volume útil de $8,7 \mathrm{~m}^{3}$ e tempo de detenção hidráulica de 14 h).

Os quatro sistemas alagados foram construídos em alvenaria de 1,0 m de largura, $24 \mathrm{~m}$ de comprimento e $0,30 \mathrm{~m}$ de altura, em relação à base, do meio suporte e fundo plano. Os tanques foram impermeabilizados com lona de PVC $(0,5 \mathrm{~mm}$ de espessura) e, como meio suporte, foi utilizada a brita \# 0 (volume de vazios $48,4 \%)$. Na saída dos SACs foram instaladas tubulações reguladoras do nível do líquido no meio poroso, que foi mantido em $25 \mathrm{~cm}$ de altura.

Após o plantio das mudas de lírio amarelo, os leitos dos SACs foram preenchidos até a altura de $25 \mathrm{~cm}$ com água proveniente do Ribeirão São Bartolomeu, para iniciar a adaptação das plantas ao meio suporte (Figura 1). Quinze dias após o plantio, os SACs foram esgotados, sendo então preenchidos com efluente primário de esgoto doméstico, assim permanecendo durante 60 dias, com a finalidade de adaptação das plantas ao novo meio suporte e ocorrer a formação do biofilme. Após este período de adaptação do sistema iniciouse a aplicação diária de esgoto nas taxas predefinidas, dando início à fase experimental de monitoramento.

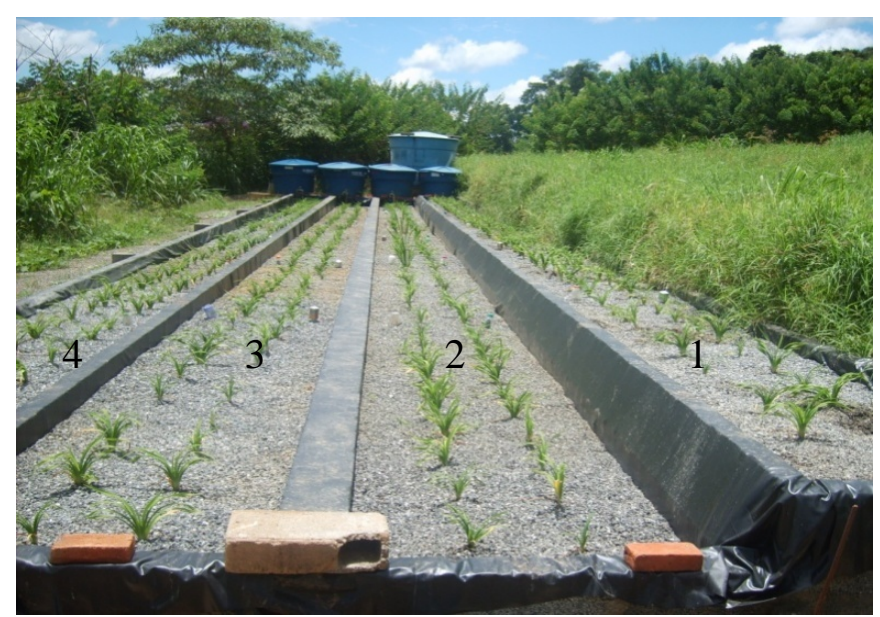

Figura 1. Aspecto do desenvolvimento inicial das mudas de lírio amarelo (Hemerocallis flava) nos sistemas alagados construídos (SAC)

Em todos os SACs utilizou-se o escoamento subsuperficial ( $25 \mathrm{~cm}$ de lâmina de esgoto em escoamento em meio suporte de $30 \mathrm{~cm}$ de altura). Os SACs foram, no entanto, submetidos a distintos tempos de detenção hidráulica (TDH) e qualidade do afluente, sendo o SAC 4 alimentado por esgoto bruto (EB) e os SACs 1, 2 e 3 alimentados por efluentes provenientes do tanque séptico (TS), a fim de se obter as taxas de carregamento orgânico estabelecidas para os tratamentos. As taxas de carregamento orgânico superficial (TCOs) foram de 44, 98, 230 e $395 \mathrm{~kg} \mathrm{ha}^{-1} \mathrm{~d}^{-1}$ de DBO, respectivamente, para os SACs 1, 2, 3 e 4 (Tabela 1).

Para avaliação do desempenho dos SACs na remoção dos poluentes foram coletadas amostras no início $(0 \mathrm{~m})$, no final $(24$ m) e em pontos intermediários (4, 8, 12, 16 e 20 m). Para coleta de amostras da água residuária nos pontos intermediários dos
Tabela 1. Características construtivas e operacionais dos sistemas alagados construídos (SACS)

\begin{tabular}{lcccc}
\hline \multirow{3}{*}{ Variável } & \multicolumn{4}{c}{ SAC $^{*}$} \\
\cline { 2 - 4 } & $\mathbf{1}$ & $\mathbf{2}$ & $\mathbf{3}$ & $\mathbf{4}$ \\
\cline { 2 - 4 } & \multicolumn{4}{c}{ TS } \\
Largura $(\mathrm{m})$ & 1,0 & 1,0 & 1,0 & 1,0 \\
Comprimento (m) & 24,0 & 24,0 & 24,0 & 24,0 \\
Área $\left(\mathrm{m}^{2}\right)$ & 24,0 & 24,0 & 24,0 & 24,0 \\
$\mathrm{~h}^{(1)}(\mathrm{m})$ & 0,25 & 0,25 & 0,25 & 0,25 \\
$\operatorname{TDH}^{(2)}(\mathrm{d})$ & 3,9 & 2,0 & 1,0 & 0,75 \\
$\operatorname{Vazão}^{2}\left(\mathrm{~m}^{3} \mathrm{~d}^{-1}\right)$ & 0,74 & 1,45 & 2,9 & 3,8 \\
$\operatorname{TCO}^{(3)}\left(\mathrm{kg} \mathrm{ha}^{-1} \mathrm{~d}^{-1}\right)$ & 44,0 & 98,0 & 230,0 & 395,0 \\
\hline
\end{tabular}

(1)h - Altura de lâmina de es goto; ${ }^{(2)} \mathrm{TDH}$ - Tempo de detenção hidráulica; ${ }^{(3)} \mathrm{TCO}$ - Taxa de carregamento orgânico

* TS - tanque séptico; EB - esgoto bruto

SACs foram inseridos, lado a lado, tubos de PVC de $50 \mathrm{~mm}$ de diâmetro, perfurados em toda a sua extensão, aprofundados até o fundo do tanque e posicionados a cada $4 \mathrm{~m}$ de distância uns dos outros. Visando à retirada das amostras, utilizou-se uma seringa de $500 \mathrm{~mL}$ com a qual era recolhida a amostra da água residuária contida no interior do tuboe; em seguida realizou-se, no Laboratório de Qualidade da Água da UFV, a análise de DQO (Demanda Química de Oxigênio), utilizando-se o método de oxidação química em refluxo aberto (APHA et al., 2005).

Os dados de DQO obtidos em amostras coletadas ao longo do comprimento dos SACs foram utilizados para ajuste do modelo matemático proposto por Brasil et al. (2007b), que é uma adaptação ao modelo de remoção de primeira ordem, conforme apresentado na Eq.1:

$$
\frac{\mathrm{C}}{\mathrm{C}_{0}}=\mathrm{e}^{\mathrm{K}_{\mathrm{V}} \cdot \mathrm{t}^{\mathrm{n}}}
$$

em que:

C - concentração de efluente, $\mathrm{mg} \mathrm{L}^{-1}$

$\mathrm{C}_{0}$ - concentração de afluente, $\mathrm{mg} \mathrm{L}^{-1}$

$\mathrm{K}_{\mathrm{v}}$ - coeficiente de decaimento do poluente, $\mathrm{d}^{-1}$

t - tempo de detenção hidráulica, $d$

n - coeficiente associado ao aumento da resistência à degradação do material orgânico remanescente e às alterações hidrodinâmicas no sistema, adimensional

\section{RESULTADOS E DISCUSSÃO}

Na Figura 2 estão os resultados da estatística descritiva relativa à concentração de DQO na água residuária em tratamento, ao longo do comprimento dos SACs.

As eficiências na remoção de DQO nos SACs 1, 2, 3 e 4 foram, respectivamente, 72,1;63,7; 72,2 e 70,0\%.

Abrahão (2006) observou, ao tratar água residuária de laticínios em SACs cultivados com capim tifton-85 e capim elefante, aumento da capacidade do sistema em remover DQO, a despeito do aumento da TCO e o atribuiu ao provável acúmulo de material particulado no sistema, tendo este material funcionado como filtro tornando possível maior remoção de 

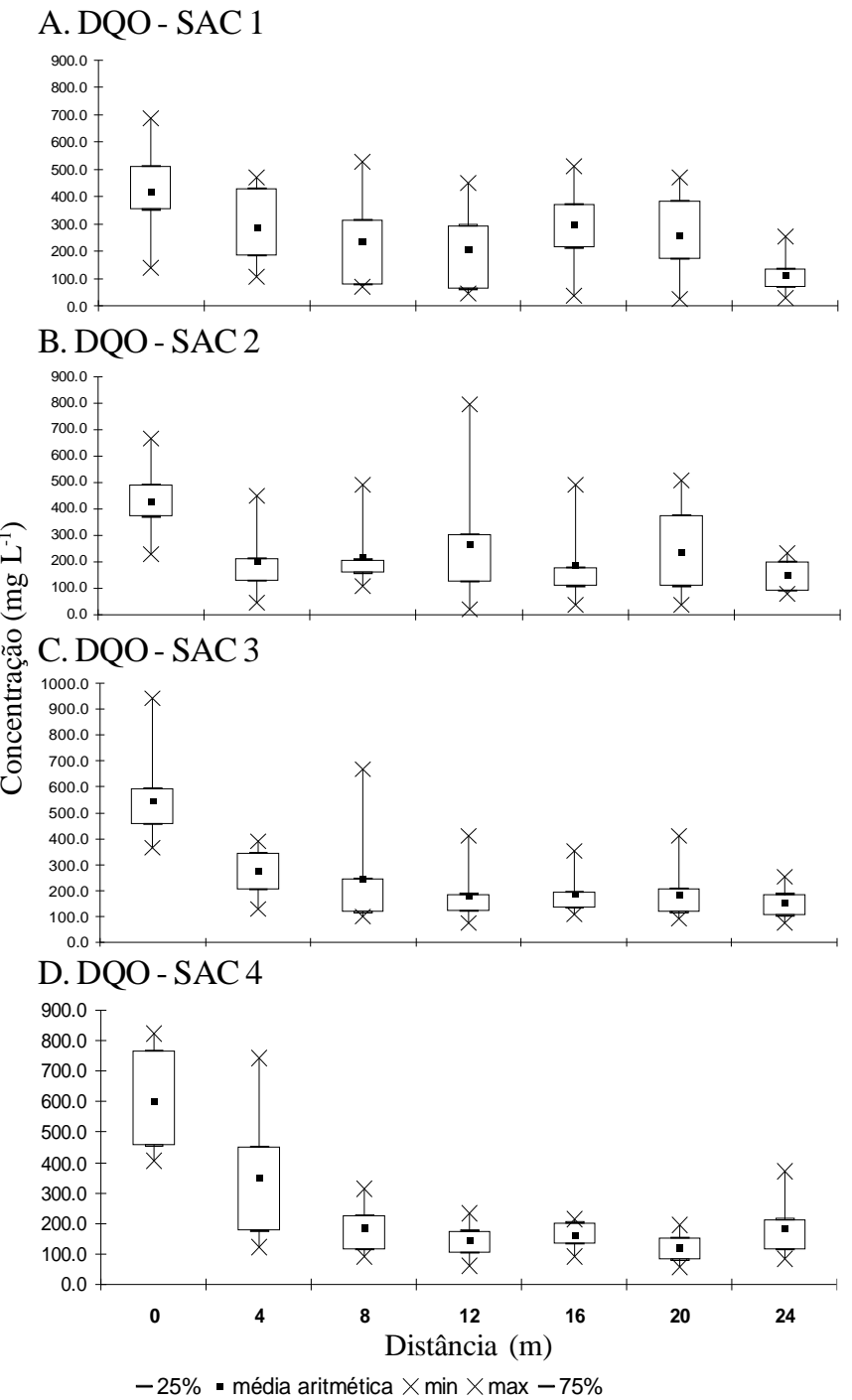

Figura 2. Decaimento da $\mathrm{DQ} O$ na água residuária em tratamento ao longo do comprimento dos sistemas alagados construídos (SACs)

DQO particulada. Além disso, o autor suspeitou que a intensificação na formação do biofilme no meio suporte proporcionada com o aumento nas TCO, também possa ter aumentado a capacidade do sistema em remover DQO.

Eficiências semelhantes na remoção de DQO foram obtidas por Matos et al. (2010a) no tratamento de água residuária de suinocultura, além de remoção mínima de DQO de $87 \%$, em SAC cultivado com Alternanthera, e máxima de $92 \%$, em SAC misto (cultivado com taboa, Alternanthera e capim tifton 85). Da mesma forma, Brasil et al. (2005) obtiveram eficiências na remoção de DQO entre 81 e $85 \%$ em SACs cultivados com taboa, no tratamento de água residuária doméstica. Valentim (2003) obteve, assim, neste trabalho, eficiência de 70\% na remoção de DQO no tratamento de água residuária doméstica em SACs cultivados com taboa. Fia (2009) obteve remoção de 66 a 80 e de 69 a $79 \%$ na DQO em SACs cultivados com capim tifton-85 e taboa, respectivamente, no tratamento de água residuária da suinocultura. Comparando os resultados obtidos com os de outros trabalhos em que os SACs foram cultivados com plantas ornamentais, observa-se maior eficiência do sistema cultivado com lírio amarelo. Zurita et al. (2009) obtiveram entre 76 e 79,7\% de remoção na DBO e 75,5 e 77, $1 \%$ na de DQO de esgoto doméstico tratado em SACs cultivados com copode-leite e em sistema misto (cultivado com ave do paraíso, antúrio e agapanto), respectivamente.

Nas Figuras 3A, 3B, 3C e 3D se encontram os resultados dos ajustes dos modelos de remoção de matéria orgânica aos dados experimentais de cada SAC e, na Figura 3e, o ajuste obtido quando se utilizaram os dados conjuntos de todos os SACs. Aidéia do ajuste de uma única equação é a de possibilitar a obtenção de uma equação única capaz de representar a cinética de decaimento do material orgânico para qualquer uma das TCOs aplicadas. Verifica-se, ao se avaliar o ajuste da equação geral que, com o modelo proposto por esses autores, pode-se descrever adequadamente a cinética de remoção de matéria orgânica (DQO) de água residuária doméstica, podendo ser utilizado para estimativa da concentração relativa, na faixa de TCO avaliada, quando do seu tratamento em SACs. Fia (2009), ao avaliar diferentes modelos matemáticos (Brasil et al., 2007b; Cooper, 1999; Kadlec \& Knight, 1996; Laber et al., 1999) na descrição da cinética de degradação da matéria orgânica em SACs cultivados com taboa e capim tifton- 85 no tratamento de água residuária da suinocultura, também notou que dentre os modelos avaliados aquele proposto por Brasil et al. (2007b) e por Kadlec \& Knight (1996) foi o que apresentou o melhor ajuste (maior $\mathrm{R}^{2}$ ) aos dados coletados no experimento, tal como observado por Vitali et al. (2008), Fia et al. (2009) e Matos et al. (2010c). Os ajustes apresentados pelo modelo de Laber et al. (1999) e Cooper (1999) se aproximaram aos ajustes obtidos pela equação de primeira ordem, apresentando baixos valores de $\mathrm{R}^{2}$ quando comparados com os modelos apresentados por Brasil et al. (2007b) e por Kadlec \& Knight (1996), mostrandose menos adequado para descrever a cinética de degradação de DQO, de acordo com os dados obtidos neste trabalho.

No ajuste da equação adaptada de cinética de primeira ordem na remoção da matéria orgânica nos SACs (Brasil et al., 2007b) foram obtidos os valores de $0,45 \mathrm{~d}^{-1} ; 0,69 \mathrm{~d}^{-1} ; 1,23 \mathrm{~d}^{-1}$ e $1,72 \mathrm{~d}^{-1}$ para $\mathrm{K}_{\mathrm{v}}$ nos SACs 1, 2, $3 \mathrm{e} 4$, respectivamente. Os coeficientes de decaimento do material orgânico obtidos nos dois primeiros SACs podem ser considerados baixos quando comparados aos apresentados na literatura para diferentes águas residuárias. Fia (2009), ao aplicar taxas de carregamentos orgânicos crescentes (519; $1.059 ; 1.477$ e $1.997 \mathrm{~kg} \mathrm{ha}^{-1} \mathrm{~d}^{-1}$ de DQO) em SACs cultivados com capim tifton-85 no tratamento de águas residuárias da suinocultura obteve valores de coeficiente de decaimento da matéria orgânica de 1,$35 ; 1,54 ; 1,47{\mathrm{e} 1,51 \mathrm{~d}^{-1}}^{-1}$ para TDHs de 12; 5,9; 4,2 e 3,2 d, respectivamente; já em SACs cultivados com taboa, que receberam taxas de $526 ; 1.051 ; 1.477$ e $1.652 \mathrm{~kg} \mathrm{ha}^{-1} \mathrm{~d}^{-1}$ de DQO ele obteve, respectivamente, valores de: 1,$31 ; 1,51$ e 1,64 d $\mathrm{d}^{-1}$ para TDHs de 11,$8 ; 5,9 ; 4,2$ e 4,0 d. É de bom alvitre se considerar que a espécie cultivada e a época (estação) do ano possam interferir na eficiência de degradação da matéria orgânica presente nas águas residuárias em tratamento em SACs. Ao utilizarem SACs cultivados com taboa, tripa de sapo, tifton-85 e SAC misto (as três espécies em sequência), no tratamento de águas residuárias da suinocultura, Matos et al. (2010c), não observaram diferença significativa no coeficiente de decaimento da matéria orgânica quando aplicaram uma TCO de 590,9 $\mathrm{kg} \mathrm{ha}^{-1} \mathrm{~d}^{-1}$ de DQO, o que 
A.

B.

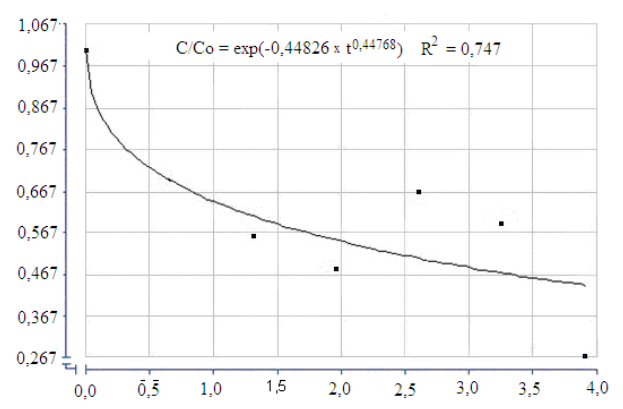

C.

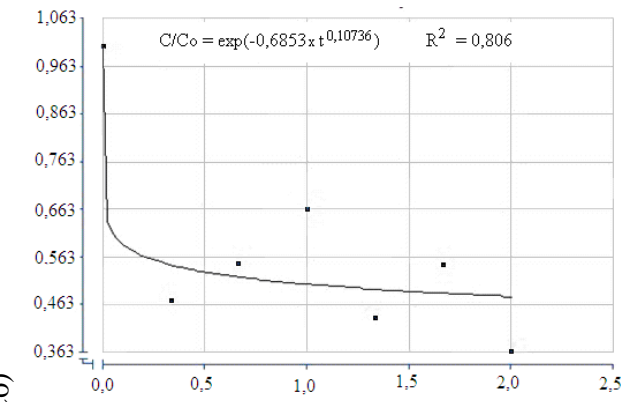

0
0
0
0
0
0
0
0
0
0
0

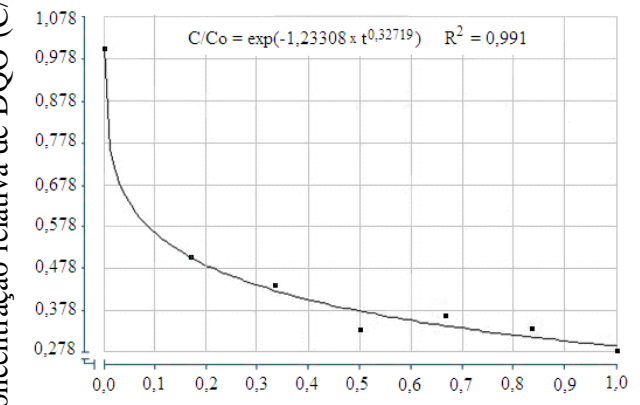

D.

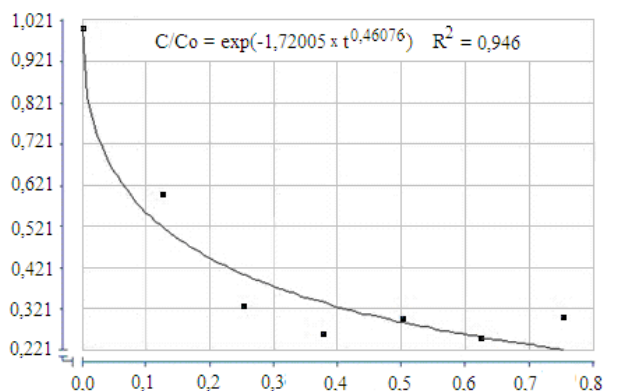

E

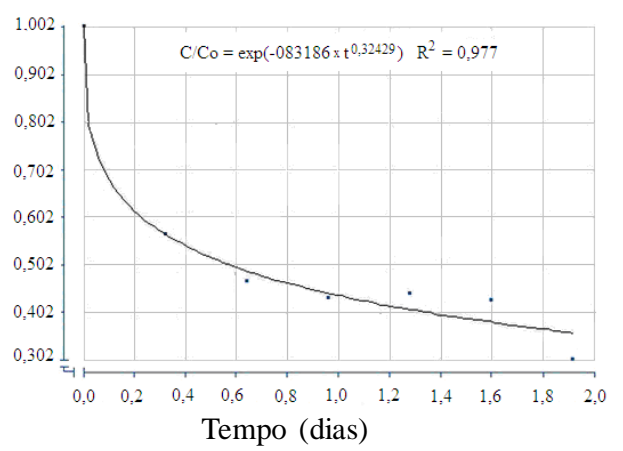

Figura 3. Ajustes do modelo matemático adaptado de remoção de primeira ordem para remoção da matéria orgânica da água residuária doméstica nos sistemas alagados construídos (SACs): 1 (A), 2 (B), 3 (C), 4 (D) e em (E) está apresentada a curva ajustada quando foram utilizados dados obtidos em todos os SACs correspondeu a um TDH de 4,8 d obtendo-se, no período de verão, valores de $\mathrm{K}_{\mathrm{v}}$ de 1,$52 ; 0,94 ; 1,39$ e $1,44 \mathrm{~d}^{-1}$, respectivamente; no período do inverno os autores obtiveram valores de 0,$88 ; 1,07 ; 0,78$ e $0,96 \mathrm{~d}^{-1}$, respectivamente. Como a aplicação da água residuária neste trabalho foi realizada no período de inverno/primavera, observa-se que o coeficiente de degradação obtido no SAC $4\left(1,72 \mathrm{~d}^{-1}\right)$, o qual recebeu carga de $395 \mathrm{~kg} \mathrm{ha}^{-1} \mathrm{~d}^{-1}$, foi substancialmente maior que os obtidos por Matos et al. (2010c) e Fia (2009). Suspeita-se que esses resultados possam estar associados a uma possível maior degradabilidade do material orgânico presente no esgoto doméstico, tendo em vista se encontrar na sua parte como material orgânico solúvel e menos na forma particulada, como é o caso da água residuária da suinocultura.

Brasil et al. (2007b) obtiveram, ao trabalhar com água residuária doméstica, o seguinte ajuste da equação modificada por eles, para a cinética de remoção de DQO: $\mathrm{C} / \mathrm{C}_{0}=$ $\exp \left(1,6221 \cdot \mathrm{t}^{0.241}\right) \operatorname{com} \mathrm{R}^{2}=0,981$, ou seja, valor de $\mathrm{K}_{v}$ de $1,62 \mathrm{~d}^{-1}$, valor bastante próximo ao obtido neste trabalho no SAC que recebeu a maior carga orgânica $\left(1,72 \mathrm{~d}^{-1}\right)$. Os mesmos autores geraram a equação $C / C_{0}=\exp \left(-0,833 . t^{0,356}\right) \operatorname{com} R^{2}=0,999$, ao utilizarem os dados de remoção de DQO obtidos na pesquisa de Valentim (2003), que trabalhou com o mesmo tipo de água residuária. Como se pode observar na Figura $3 \mathrm{E}$, ao se utilizar a equação média (ajustada com os dados obtidos em todos os SACs), ajustaram-se valores de $\mathrm{K}_{\mathrm{v}}$ e "n" semelhantes aos obtidos por Valentim (2003), cuja equação: $C / C_{0}=\exp (-$ $0,832 . .^{0,324}$ ) apresentou $R^{2}=0,977$ considerando-se, porém, apenas as curvas ajustadas com $R^{2}$ maior que 0,90 , verifica-se que a equação ajustada se aproxima mais à obtida por Brasil et al. (2007b).

Em geral, os SACs com menor TCO apresentaram também menor coeficiente de decaimento de matéria orgânica $\left(\mathrm{K}_{v}\right)$, fato que se deve, sem dúvida, à menor disponibilidade de matéria orgânica degradável no meio. Considerando-se que neste experimento menor TCO representou maior TDH, verificou-se que os SACs sinalizaram grande capacidade de amortização das cargas orgânicas aplicadas já que a eficiência pouco alterou com a TCO aplicada. Em geral ocorreuu, nos SACs submetidos aos maiores TDHs, redução nos valores dos coeficientes de decaimento quando comparados aos SACs com menor TDH, tal como observado por Fia (2009). Freitas (2006) também verificou que $o$ aumento na taxa de carregamento proporcionou a obtenção de maiores valores de $\mathrm{K}_{\mathrm{v}}$. $\mathrm{O}$ autor considerou que se os SACs utilizados em seu experimento tivessem a metade do tamanho utilizado, ou seja, tivessem seu TDH reduzido, mantida a mesma TCO, os valores de $\mathrm{K}_{\mathrm{v}}$ obtidos seriam maiores e comparáveis aos obtidos na literatura. Além disso, pela própria definição da reação de primeira ordem a taxa de decaimento é proporcional à concentração do reagente que, neste caso, é a DQO. Assim, maiores taxas de carregamento orgânico resultam em também maiores valores de $\mathrm{K}_{v}$.

$\mathrm{Na}$ interpretação dos valores dos parâmetros ajustado é preciso levar em consideração que, quanto maior o valor de $\mathrm{K}_{\mathrm{v}}$ maior foi o decréscimo na relação $\mathrm{C} / \mathrm{C}_{0}$ no início dos $\mathrm{SACs}$, o que repercutiu em maior eficiência na remoção de DQO, quando o modelo hidráulico de fluxo em pistão explicou adequadamente o comportamento dessa variável ao longo do sistema. No SAC2 
aparentemente modelos hidráulicos de dispersão e mistura completa poderiam ser mais adequados, já que neste SAC houve grande remoção do início, seguido de certa instabilidade nos valores de DQO ao longo do sistema. Desta forma, fugiu à tendência ocorrida nos outros SACs de que maiores remoções iniciais levariam a maior remoção global do sistema.

Quanto aos valores de “n”, foram obtidos valores de 0,448 ; 0,107; 0,327 e 0,461 para os SACs 1, 2, 3 e 4. Tal como já apresentado, este parâmetro cinético está associado à presença do aumento relativo na quantidade de material orgânico mais resistente à degradação ao longo do comprimento dos SACs e às alterações nas condições hidrodinâmicas do sistema, sendo uma das razões a evapotranspiração no meio (Brasil et al., 2007b). Quanto maior seu valor maior a taxa de remoção de DQO, notadamente a partir dos primeiros $4 \mathrm{~m}$ do início dos SACs, o que também conduz a uma eficiência maior do sistema na remoção do material orgânico da água residuária em tratamento, naqueles SACs em que o modelo hidráulico de fluxo em pistão descreveu adequadamente o comportamento dos dados. Novamente em função da maior dispersão ocorrida no SAC2, o que foi descrito acima não foi confirmado. Matos et al. (2010c) obtiveram valores de 0,07;0,52;0,30 e 0,20 em SACs cultivados com taboa, tripa-de-sapo, capim tifton-85 e SAC misto, respectivamente, no tratamento de águas residuárias da suinocultura, no período do verão; no inverno os autores obtiveram valores de 0,38; 0,32; 0,63 e 0,53 nos mesmos SACs. Brasil et al. (2007b) obtiveram, ao trabalhar com água residuária doméstica, 0,25 e, ao utilizar os dados de remoção de DQO obtidos na pesquisa de Valentim (2003), geraram a equação proposta por eles, obtendo o valor de 0,356 para $n$.

\section{ConClusõES}

1. O modelo proposto por Brasil et al. (2007b) indicou bom ajuste e descreveu adequadamente a cinética de remoção de matéria orgânica (DQO) nos sistemas avaliados apresentando, geralmente, $\mathrm{R}^{2}$ superior a $75 \%$, tendo sido ajustada a equação geral $\mathrm{C} / \mathrm{C}_{0}=\exp \left(-0,832 \cdot \mathrm{t}^{0,324}\right) \operatorname{com~R}^{2}=0,977$;

2. Os sistemas alagados construídos (SACs) 1, 2, 3 e 4, com taxas de carregamento orgânico de 44, 98, 230 e $395 \mathrm{~kg} \mathrm{ha}^{-1} \mathrm{~d}^{-1}$, respectivamente, apresentaram valores de coeficientes de decaimento da DQO $\left(\mathrm{K}_{\mathrm{v}}\right)$ de $0,45 \mathrm{~d}^{-1} ; 0,69 \mathrm{~d}^{-1} ; 1,23 \mathrm{~d}^{-1}$ e $1,72 \mathrm{~d}^{-1}$, considerados baixos para os dois primeiros SACs e normais para os outros dois além de valores de "n" de 0,448; 0,107; 0,327 e 0,461 , sendo esta a faixa normalmente encontrada na literatura.

\section{LITERATURA CITADA}

Abrahão, S. S. Tratamento de água residuária de laticínios em sistemas alagados construídos cultivados com forrageira. Viçosa: UFV, 2006. 110p. Dissertação Mestrado

APHA - American Public Health Association; AWWA - American Water Works Association; WEF - Water Environment Federation. Standard methods for the examination of water and wastewater. 21.ed. Washington: APHA/AWWA/WEF, 2005. 1268p.
Belmont, M. A.; Metcalfe, C. D. Feasibility of using ornamental plants (Zantedeschia aethiopica) in subsurface flow treatment wetlands to remove nitrogen, chemical oxigen demand and nonylphenol ethoxylate surfactants - a laboratory-scale study. Ecological Engineering, v.21, p.233247, 2003.

Brasil, M. S.; Matos, A. T. Avaliação de aspectos hidraúlicos e hidrológicos de sistemas alagados construídos de fluxo subsuperficial. Engenharia Sanitária e Ambiental, v.13, p.323328, 2008.

Brasil, M. S.; Matos, A.T.; Silva, C. M.; Cecon, P.R.; Soares, A. A. Modeling of pollution removal in constructed wetlands with horizontal subsurface flow. Agricultural Engineering Research, v.13, p.48-56, 2007a.

Brasil, M. S.; Matos, A. T.; Soares, A. A. Plantio e desempenho fenológico da taboa (Thypha sp.) utilizada no tratamento de esgoto doméstico em sistema alagado construído. Revista Engenharia Sanitária e Ambiental, v.12, p.266-272, 2007 b.

Brasil, M. S.; Matos, A. T.; Soares, A. A.; Ferreira, P. A. Qualidade do efluente de sistemas alagados construídos, utilizados no tratamento de esgoto doméstico. Revista Brasileira de Engenharia Agrícola e Ambiental, v.9, p.133$137,2005$.

Carleton, J. N. Damköhler distributions and constituent removal in treatment wetlands. Ecological Engineering, v.19, p.233248, 2002.

Cooper, P. A review of the design and performance of verticalflow and hybrid reed bed treatment systems. Water Science and Technology, v.40, p.1-9, 1999.

Eustáquio Júnior, V.; Matos, A. T.; Campos, L. C.; Borges, A. C. Desempenho agronômico da aveia preta (Avena strigosa Schreb.) cultivada em sistemas alagados construídos. AmbiÁgua, v.05, p.68-78, 2010.

Fia, F. L. R. Modelos de remoção de variáveis qualitativas em sistemas alagados construídos utilizados no tratamento de águas residuárias da suinocultura. Viçosa: UFV, 2009. 156p. Tese Doutorado

Fia, R.; Luiz, F. A. R.; Matos, A. T.; Lambert, T. F.; Matos, M. P. Avaliação de modelos de degradação de matéria orgânica em sistemas alagados construídos. In: Congresso Brasileiro de Engenharia Agrícola, 38, 2009, Juazeiro/Petrolina. Anais... Jaboticabal: SBEA, 2009. CD-Rom

Fia, R.; Matos, A. T.; Ferreira, P. A.; Teodoro, P. E. P.; Schuery, F. C.; Luiz, F. A. R. Desempenho agronômico da thypha sp. e alternanthera philoxeroides mart utilizadas no tratamento de águas residuárias da lavagem e descascamento/despolpa dos frutos do cafeeiro em sistema alagado construído. Engenharia na Agricultura, v.16, p.436-448, 2008.

Fia, R.; Matos, A. T.; Fia, F. R. L.; Matos, M. P.; Lambert, T. F.; Nascimento, F. S. Desempenho de forrageiras em sistemas alagados de tratamento de águas residuárias do processamento do café. Revista Brasileira de Engenharia Agrícola e Ambiental, v.14, p.842-847, 2010.

Freitas, W. S. Desempenho de sistemas alagados construídos, cultivados com diferentes espécies vegetais, no tratamento de águas residuárias da suinocultura. Viçosa: UFV, 2006, 159p. Tese Doutorado 
Kadlec, R. H.; Knight, R. L. Treatment wetlands. Florida: CRC Press, 1996, 893p.

Kincanon, R.; McAnally, A. S. Enhancing commonly used model predictions for constructed wetland performance: asbuilt design considerations. Ecological Modelling, v.174, p.309-322, 2004.

Konnerup, D.; Koottatep, T.; Brix, H. Treatment of domestic wastewater in tropical, subsurface ûow constructed wetlands planted with Canna and Heliconia. Ecological engineering, v.35, p.248-257, 2009.

Laber, J.; Haberl, R.; Shrestha, R. Two-stage construted wetland for treating hospital wastewater in Nepal. Water Science and Technology, v.40, p.317-324, 1999.

Matos, A. T.; Abrahão, S. S.; Pereira, O. G. Desempenho agronômico do capim tifton 85 (Cynodon spp) cultivados em sistemas alagados construídos utilizados no tratamento de água residuária de laticínios. Ambi-Água, v.3, p.43-53, 2008.

Matos, A. T.; Freitas, W. S.; Borges, A. C. Dinâmica da remoção de matéria orgânica de águas residuárias da suinocultura em sistemas alagados construídos cultivados com diferentes espécies vegetais. In: Congresso Brasileiro de Engenharia Agrícola, 39, 2010c, Vitória. Anais...Jaboticabal: SBEA, 2010c. CD Rom.

Matos, A. T.; Freitas, W. S.; Fia, R.; Matos, M. P. Qualidade do efluente de sistemas alagados construídos utilizados no tratamento de águas residuárias da suinocultura visando seu reuso. Engenharia na Agricultura, v.17, p.383-391, 2009a.

Matos, A. T.; Freitas, W. S.; Lo Monaco, P. A.V. Eficiência de sistemas alagados construídos na remoção de poluentes de águas residuárias da suinocultura. Ambi-Água, v.5, p.119132, 2010a.

Matos, A. T.; Freitas, W. S.; Lo Monaco, P. A. V. Capacidade extratora de diferentes espécies vegetais cultivadas em sistemas alagados utilizados no tratamento de águas residuárias da suinocultura. Ambi-Água, v.04, p.31-45, 2009 b.
Matos, A. T., Freitas, W. S.; Martinez, M. A.; Tótola, M. R.; Azevedo, A. A. Tifton grass yield on constructed wetland used for swine wastewater treatment. Revista Brasileira de Engenharia Agrícola e Ambiental, v.14, p.510-516, 2010 b.

Reed, S. C.; Crites, R. W.; Middlebrooks, E. J. Natural systems for management and treatment. New York: McGraw-Hill, Inc. 1995, 435p.

Rousseau, D. P. L.; Vanrolleghem, P. A.; Pauw, N. D. Modelbased design of horizontal subsurface flow constructed treatment wetlands: A review. Water Research, v.38, p.14841493, 2004.

Valentim, M. A. A. Desempenho de leitos cultivados ("construted wetland") para tratamento de esgoto: contribuições para concepção e operação. Campinas: UNICAMP/FEAGRI, 2003. 210p. Tese Doutorado

Vitalli, G. L. S.; Olijnyk, D. P.; Santos, D. C.; Philippi, L. S. Evaluation of modelling methods for the constructed wetland design. In: Congreso Interamericano de Ingeniería Sanitaria y Ambiental, 31, 2008, Santiago, Chile. Proceedings... Santiago, Chile: AIDS, 2008. CD-Rom

Wang, R.; Korboulewsky, N.; Prudent, P.; Baldy, V.; Bonin, G. Can vertical-flow wetland systems treat high concentrated sludge from a food industry? A mesocosm experiment testing three plant species. Ecological Engineering, v.35, p.230-237, 2009.

Wood, A. Constructed wetlands in water pollution control: fundamentals to their understanding. Water Science and Tecnology, v.32, p.21-29, 1995.

Zurita, F.; Anda, J. D.; Belmont, M. A. Treatment of domestic wastewater and production of commercial flowers in vertical and horizontal subsurface-flow constructed wetlands. Ecological Engineering, v.35, p.861-869, 2009.

Zurita, F.; Belmont, M.A.; Anda, J. D.; Martinez, J. C. Stress detection by laser-induced fluorescence in Zantedeschia aethiopica planted in subsurface-flow treatment wetlands. Ecological Engineering, v.33, p.110-118, 2008. 